\title{
THE MAGNETIC BOSE LIQUID
}

\author{
I. O. Vakarchuk \\ Ivan Franko National University of Lviv, Department for Theoretical Physics \\ 12 Drahomanov Str., Lviv, UA-79005, Ukraine
}

(Received May 21, 2002)

\begin{abstract}
We study a quantum liquid which consists of Bose-particles each carrying a magnetic moment. The thermodynamic and structure functions of the system are calculated in the random phase approximation using functional integration techniques. The obtained expressions are valid in the low-temperature regime and recover the known results of the classical magnetic liquids theory as a limiting case. In particular, the influence of the magnetic degrees of freedom as well as of the external magnetic field on the phenomenon of Bose-condensation is studied.
\end{abstract}

Key words: spin-polarized hydrogen, Bose-Einstein condensation, magnetic liquid.

PACS number(s): 03.75.Fi, 67.65.+z, 75.50.Mm

\section{INTRODUCTION}

In the wake of the experimental study of the properties of the atomic polarized hydrogen [1-3] an interesting object of theoretical study can be found in the magnetic Bose-liquid whose model with the preliminary results was published by us in [4]. In what follows we will present the sequential solution of the problem.

We will consider in the volume $V$ a set of Bose-particles $N$ with the mass $m$ and the integer spin $s$ with the Hamiltonian $\hat{H}$ consisting of the sum of the ordinary liquid Hamiltonian $\hat{H}_{B}$ and the magnetic part of the Hamiltonian $\hat{H}_{s}$ describing the Heisenberg exchange interaction between the spins and their interaction with the external magnetic field of strenght $\mathcal{H}$ :

$$
\hat{H}=\hat{H}_{B}+\hat{H}_{s}
$$

The liquid Hamiltonian

$$
\hat{H}_{B}=\sum_{j=1}^{N} \frac{\hat{\mathbf{p}}_{j}^{2}}{2 m}+\sum_{1 \leq i<j \leq N} \Phi\left(\left|\mathbf{r}_{i}-\mathbf{r}_{j}\right|\right)
$$

consists of the sum of the kinetic energy operator of the particles and the potential energy of the pair interaction between them, here $\mathbf{r}_{j}$ and $\hat{\mathbf{p}}_{j}$ are the coordinate and the momentum operator of the $j$-th particle; $\Phi\left(\left|\mathbf{r}_{i}-\mathbf{r}_{j}\right|\right)$ is he interaction energy between the $i$-th and $j$-th particles. At the same time we suppose that the bound states between pairs of particles are absent. The spin Hamiltonian

$$
\hat{H}_{s}=-\sum_{1 \leq i<j \leq N} J\left(\left|\mathbf{r}_{i}-\mathbf{r}_{j}\right|\right) \hat{\mathbf{s}}_{i} \hat{\mathbf{s}}_{j}-\mu \mathcal{H} \sum_{j=1}^{N} \hat{s}_{j}^{z},
$$

where the $j$-th particle spin operator $\hat{\mathbf{s}}_{j}=\left(\hat{s}_{j}^{x}, \hat{s}_{j}^{y}, \hat{s}_{j}^{z}\right), \mu$ is its magnetic moment, $J\left(\left|\mathbf{r}_{i}-\mathbf{r}_{j}\right|\right)$ is the energy of the exchange interaction between the $(i, j)$-pair of particles, the external magnetic field will be directed along the $z$ axis, $\mathcal{H}=(0,0, \mathcal{H})$.

Our task lies in finding the thermodynamic and structure functions of such a system. The calculations will be carried out in the random phase approximation. We will be equally concerned with the influence of the magnetic field on the properties of the superfluid Bose liquid and with the change of its magnetic characteristics as a consequence of the fact that the motion of liquid atoms abides by the quantum laws. In the classical limit $\hbar \rightarrow 0$ for the liquid subsystem we will obtain the appropriate expressions for the classical magnetic liquid found in [5].

\section{INITIAL EQUATION}

In order to calculate the thermodynamic quantities it is necessary to calculate the partition function

$$
Z_{N}=\mathrm{Sp} e^{-\beta \hat{H}}
$$

where the inverse temperature $\beta=1 / T, T$ is the system's temperature, the trace being taken over all the degrees of freedom, both liquid and spin ones.

By making use of the Fourier transformations for the particles' potential energy and their exchange energy we will start with rewriting the Hamiltonian (1.1) through the density and spin fluctuations. Thus for (1.2) we will have

$$
\begin{aligned}
\hat{H}_{B} & =\sum_{j=1}^{N} \frac{\hat{\mathbf{p}}_{j}^{2}}{2 m}+\frac{N(N-1)}{2 V} \nu_{0} \\
& +\frac{N}{2 V} \sum_{\mathbf{k} \neq 0} \nu_{k}\left(\rho_{\mathbf{k}} \rho_{-\mathbf{k}}-1\right),
\end{aligned}
$$

where the Fourier coefficient of the potential energy of two particles 


$$
\nu_{k}=\int e^{-i \mathbf{k} \mathbf{R}} \Phi(R) d \mathbf{R}
$$

and the quantity

$$
\rho_{\mathbf{k}}=\frac{1}{\sqrt{N}} \sum_{j=1}^{N} e^{-i \mathbf{k r}_{j}}
$$

is the Fourier coefficient of the particle density fluctuations. The components of the wave vector $\mathbf{k}$ run through the integer values which are multiple to $2 \pi / V^{1 / 3}$ and when the volume $V \rightarrow \infty$ the sum over $\mathbf{k}$ becomes the integral $\sum_{\mathbf{k}}=N \int d \mathbf{k} /(2 \pi)^{3}$.

In the same fashion we find that for the spin Hamiltonian (1.3)

$$
\begin{aligned}
\hat{H}_{s} & =\frac{N}{2 V} s(s+1) \sum_{\mathbf{k}} J_{k}-\frac{N}{2 V} \sum_{\mathbf{k}} J_{k} \hat{\mathbf{S}}_{\mathbf{k}} \hat{\mathbf{S}}_{-\mathbf{k}} \\
& -\mu \mathcal{H} \sum_{j=1}^{N} \hat{s}_{j}^{z},
\end{aligned}
$$

where

$$
J_{k}=\int e^{-i \mathbf{k} \mathbf{R}} J(R) d \mathbf{R}
$$

and the operator

$$
\hat{\mathbf{S}}_{\mathbf{k}}=\frac{1}{\sqrt{N}} \sum_{j=1}^{N} e^{-i \mathbf{k r}_{j}} \hat{\mathbf{s}}_{j} .
$$

In the expression for the partition function we will first sum over the spin degrees of freedom. With this purpose using (2.5) we will present the statistical operator as

$$
\begin{aligned}
e^{-\beta \hat{H}} & =e^{-\beta\left(\hat{H}_{B}+\hat{H}_{0}\right)} \exp \left(-\beta \frac{N}{2 V} s(s+1) \sum_{\mathbf{k}} J_{k}\right) \\
& \times \hat{T}_{\beta} \exp \left(\int_{0}^{\beta} d \beta^{\prime} \frac{N}{2 V} \sum_{\mathbf{k}} J_{k} \hat{\mathbf{S}}_{\mathbf{k}}\left(\beta^{\prime}\right) \hat{\mathbf{S}}_{-\mathbf{k}}\left(\beta^{\prime}\right)\right)
\end{aligned}
$$

where $\hat{H}_{0}$ denotes the Hamiltonian of the interaction of spins with the external field

$$
\hat{H}_{0}=-\mu \mathcal{H} \sum_{j=1}^{N} \hat{s}_{j}^{z},
$$

and the operator

$$
\hat{\mathbf{S}}_{\mathbf{k}}\left(\beta^{\prime}\right)=e^{\beta^{\prime}\left(\hat{H}_{B}+\hat{H}_{0}\right)} \hat{\mathbf{S}}_{\mathbf{k}} e^{-\beta^{\prime}\left(\hat{H}_{B}+\hat{H}_{0}\right)}
$$

$$
=\frac{1}{\sqrt{N}} \sum_{j=1}^{N} e^{-i \mathbf{k} \mathbf{r}_{j}\left(\beta^{\prime}\right)} e^{\beta^{\prime} \hat{H}_{0}} \hat{\mathbf{s}}_{j} e^{-\beta^{\prime} \hat{H}_{0}},
$$

where

$$
\mathbf{r}_{j}\left(\beta^{\prime}\right)=e^{\beta^{\prime} \hat{H}_{B}} \mathbf{r}_{j} e^{-\beta^{\prime} \hat{H}_{B}}
$$

The time-ordering operator $\hat{T}_{\beta}$ in (2.7) orders the operators $\hat{\mathbf{S}}_{\mathbf{k}}\left(\beta^{\prime}\right)$ appearing in the series expansion of the exponent, from the right side leftwards according to the descending temperature time $\beta^{\prime}$.

Let us write the operator under the $\hat{T}_{\beta}$ product in $(2.7)$ as a functional integral [6]:

$$
\begin{aligned}
e^{-\beta \hat{H}} & =e^{-\beta\left(\hat{H}_{B}+\hat{H}_{0}\right)} \exp \left(-\beta \frac{N}{2 V} s(s+1) \sum_{\mathbf{k}} J_{k}\right) \\
& \times \int(d \varphi) e^{-\frac{1}{2} \sum_{q} \boldsymbol{\varphi}_{q} \boldsymbol{\varphi}_{-q}} \\
& \times \hat{T}_{\beta} \exp \left(\sum_{q} \boldsymbol{\varphi}_{q} \hat{\boldsymbol{\sigma}}_{q} \sqrt{\beta N J_{k} / V}\right)
\end{aligned}
$$

the integration here going over the real $\varphi_{q}^{\prime}$ and imaginary $\varphi_{q}^{\prime \prime}$ parts of the vector variable components $\boldsymbol{\varphi}_{q}=$ $\left(\varphi_{q}^{x}, \varphi_{q}^{y}, \varphi_{q}^{z}\right)$ in the infinite limits

$$
\begin{aligned}
\int(d \varphi) & =\prod_{l=(x, y, z)} \int_{-\infty}^{\infty} \frac{d \varphi_{0}^{l}}{\sqrt{2 \pi}} \\
& \times \prod_{q \neq 0}^{\prime} \int_{-\infty}^{\infty} \frac{d \varphi_{q}^{l^{\prime}}}{\sqrt{\pi}} \int_{-\infty}^{\infty} \frac{d \varphi_{q}^{l^{\prime \prime}}}{\sqrt{\pi}}
\end{aligned}
$$

the 4 -vector $q \equiv(\mathbf{k}, \omega)$ and frequency $\omega=2 \pi n / \beta$, $n=0, \pm 1, \pm 2, \ldots$; the operator

$$
\hat{\boldsymbol{\sigma}}_{q}=\frac{1}{\beta} \int_{0}^{\beta} d \beta^{\prime} e^{i \beta^{\prime} \omega} \hat{\mathbf{S}}_{\mathbf{k}}\left(\beta^{\prime}\right)
$$

is the frequency Fourier component of the operator (2.9). The prime on the product over $q$ means that we take into account just a half space of the change of the 4-vector $q$ (this is a consequence of the fact that $\hat{\boldsymbol{\sigma}}_{q}=\hat{\boldsymbol{\sigma}}_{-q}^{*}$ ); the zero component $\boldsymbol{\varphi}_{0}$ is a real quantity.

Equation (2.10) can be checked by simple integration over $\varphi_{q}$ (as we deal here with Poisson's integrals) with the consideration for the fact that under the sign of the time-ordering operator $\hat{T}_{\beta}$ the operator is treated as an ordinary quantity.

Let us now pass on to the calculation of the trace over the spin degrees of freedom of the statistical operator $(2.7)$ : 


$$
\begin{aligned}
\operatorname{Sp}_{s} e^{-\beta \hat{H}} & =e^{-\beta \hat{H}_{B}} \exp \left(-\beta \frac{N}{2 V} s(s+1) \sum_{\mathbf{k}} J_{k}\right) \int(d \varphi) e^{-\frac{1}{2} \sum_{q} \boldsymbol{\varphi}_{q} \boldsymbol{\varphi}_{-q}} \\
& \times \operatorname{Sp}_{s}\left[e^{-\beta \hat{H}_{0}} \hat{T}_{\beta} \exp \left(\sum_{q} \boldsymbol{\varphi}_{q} \boldsymbol{\sigma}_{q} \sqrt{\beta N J_{k} / V}\right)\right]
\end{aligned}
$$

It is convenient to single out the integration over the zero component $\varphi_{0}^{z}$ in (2.12) as the operator

$$
\hat{\sigma}_{q=0}^{z}=\frac{1}{\beta} \int_{0}^{\beta} d \beta^{\prime} \frac{1}{\sqrt{N}} \sum_{j=1}^{N} e^{\beta^{\prime} \hat{H}_{0}} \hat{s}_{j}^{z} e^{-\beta^{\prime} \hat{H}_{0}}=\frac{1}{\sqrt{N}} \sum_{j=1}^{N} \hat{s}_{j}^{z}
$$

commutes with the Hamiltonian $\hat{H}_{0}$. Thus, from (2.12) we have

$$
\begin{aligned}
& \mathrm{Sp}_{s} e^{-\beta \hat{H}}=e^{-\beta \hat{H}_{B}} \exp \left(-\beta \frac{N s(s+1)}{2 V} \sum_{\mathbf{k}} J_{k}\right) \\
& \times \int_{-\infty}^{\infty} \frac{d \varphi_{0}^{z}}{\sqrt{2 \pi}} e^{-\frac{1}{2}\left(\varphi_{0}^{z}\right)^{2}} \int^{\prime}(d \varphi) e^{-\frac{1}{2} \sum_{q}^{\prime} \varphi_{q} \varphi_{-q}} \\
& \times \operatorname{Sp}\left[e^{-\beta \hat{H}_{0}^{*}} \hat{T}_{\beta} \exp \left(\sum_{q}^{\prime} \boldsymbol{\varphi}_{q} \hat{\boldsymbol{\sigma}}_{q} \sqrt{\beta N J_{k} / V}\right)\right],
\end{aligned}
$$

where the effective Hamiltonian of the interacting spins

$$
\hat{H}_{0}^{*}=\hat{H}_{0}-\varphi_{0}^{z} \sqrt{\frac{J_{0}}{\beta V}} \sum_{j=1}^{N} s_{j}^{z},
$$

or taking into account $(2.8)$

$$
\hat{H}_{0}^{*}=-\mu \mathcal{H}^{*} \sum_{j=1}^{N} s_{j}^{z},
$$

where the effective strength of the magnetic field

$$
\begin{aligned}
& \mathcal{H}^{*}=\mathcal{H}+\frac{1}{\mu} \sqrt{\frac{J_{0} N}{\beta V}} \eta, \\
& \eta=\varphi_{0}^{z} / \sqrt{N} .
\end{aligned}
$$

The primes on the symbols of the integral over $\varphi$ and of the sum over $q$ imply the absence of the quantity $\varphi_{0}^{z}$.

We will write formula (2.13) as follows:

$$
\begin{aligned}
& \operatorname{Sp}_{s} e^{-\beta \hat{H}}=e^{-\beta \hat{H}_{B}} \exp \left(-\beta \frac{N s(s+1)}{2 V} \sum_{\mathbf{k}} J_{k}\right) \sqrt{\frac{N}{2 \pi}} \int_{-\infty}^{\infty} d \eta e^{-\beta F_{0}} \int^{\prime}(d \varphi) e^{-\frac{1}{2} \sum_{q}^{\prime} \varphi_{q} \varphi_{-q}} \\
& \times\left\langle\hat{T}_{\beta} \exp \left(\sum_{q}^{\prime} \boldsymbol{\varphi}_{q} \hat{\boldsymbol{\sigma}}_{q} \sqrt{\beta N J_{k} / V}\right)\right\rangle_{0},
\end{aligned}
$$

where the free energy of a system of non-intracting spins in the effective magnetic field $\mathcal{H}^{*}$ (the so-called molecular-field approximation)

$$
\begin{aligned}
F_{0} & =\frac{N}{\beta}\left\{\frac{\eta^{2}}{2}-\ln \left[\sinh \left[\left(s+\frac{1}{2}\right) x\right] / \sinh \left(\frac{x}{2}\right)\right]\right\}, \\
x & =\beta \mu \mathcal{H}^{*},
\end{aligned}
$$

The angle brackets signifying the operation of approximation by the system described by the Hamiltonian (2.14),

$$
\langle\ldots\rangle_{0}=\frac{\operatorname{Sp}_{s}\left[e^{-\beta \hat{H}_{0}^{*}}(\ldots)\right]}{\operatorname{Sp}_{s} e^{-\beta \hat{H}_{0}^{*}}}
$$

The plan of our calculations is as follows. We will calculate the spin mean in (2.16), then integrate over the variables $\boldsymbol{\varphi}_{q}, q \neq 0$ and find the liquid effective Hamiltonian. After that we will calculate the operator trace (2.16) over the space degrees of freedom finding the full partition function dependent on the quantity $\eta$. The ultimate expression for the partition function (2.1) will be obtained after calculating the integral over the variable $\eta$ using the saddle-point method. 


\section{THE EFFECTIVE LIQUID HAMILTONIAN}

We will in (2.16) approximate the spin subsystem using the decomposition by irreducible means. We will decompose the operator (2.11) in its components

$$
\hat{\sigma}_{q}^{ \pm}=\hat{\sigma}_{q}^{x} \pm i \sigma_{q}^{y},
$$

so that now the scalar product

$$
\boldsymbol{\varphi}_{q} \hat{\boldsymbol{\sigma}}_{q}=\varphi_{q}^{-} \hat{\sigma}_{q}^{+}+\varphi_{q}^{+} \hat{\sigma}_{q}^{-}+\varphi_{q}^{z} \sigma_{q}^{z},
$$

where

$$
\varphi_{q}^{ \pm}=\frac{1}{2}\left(\varphi_{q}^{x} \pm i \varphi_{q}^{y}\right)
$$

Therefore,

$$
\begin{aligned}
\left\langle\hat{T}_{\beta} \exp \left(\sum_{q}^{\prime} \boldsymbol{\varphi}_{q} \hat{\boldsymbol{\sigma}}_{q} \sqrt{\beta N J_{k} / V}\right)\right\rangle_{0} & =\hat{T}_{\beta} \exp \left[\sum_{q} \sum_{\nu} \varphi_{q}^{\nu}\left\langle\hat{\sigma}_{q}^{\nu}\right\rangle_{0} \sqrt{\beta \frac{N}{V} J_{k}}\right. \\
& \left.+\frac{1}{2} \sum_{q_{1}} \sum_{q_{2}} \sum_{\nu_{1}} \sum_{\nu_{2}} K^{\nu_{1} \nu_{2}}\left(q_{1}, q_{2}\right) \beta \frac{N}{V} \sqrt{J_{k_{1}} J_{k_{2}}} \varphi_{q_{1}}^{\nu_{1}} \varphi_{q_{2}}^{\nu_{2}}+\ldots\right],
\end{aligned}
$$

where

$$
K^{\nu_{1} \nu_{2}}\left(q_{1}, q_{2}\right)=\left\langle\hat{T}_{\beta} \hat{\sigma}_{q_{1}}^{\nu_{1}} \hat{\sigma}_{q_{2}}^{\nu_{2}}\right\rangle_{0}-\left\langle\hat{\sigma}_{q_{1}}^{\nu_{1}}\right\rangle_{0}\left\langle\hat{\sigma}_{q_{2}}^{\nu_{2}}\right\rangle_{0}
$$

the index $\nu=(+,-, z)$, the dots in (3.2) signify the contributions which lead us beyond the random phase approximation (they will be skipped further). The external time-ordering operator (3.1) orders the particle coordinate operators $\left(\mathbf{r}_{1}, \ldots, \mathbf{r}_{N}\right)$ and the internal operator (under the sign of the mean) orders the spin operators. We will calculate the necessary means:

$$
\begin{gathered}
\left\langle\hat{\sigma}_{q}^{+}\right\rangle_{0}=\left\langle\hat{\sigma}_{q}^{-}\right\rangle_{0}=0, \\
\left\langle\hat{\sigma}_{q}^{z}\right\rangle_{0}=M_{1}(x) \frac{1}{\beta} \int_{0}^{\beta} d \beta^{\prime} e^{i \beta^{\prime} \omega} \rho_{\mathbf{k}}\left(\beta^{\prime}\right), \\
\rho_{\mathbf{k}}\left(\beta^{\prime}\right)=e^{\beta^{\prime} \hat{H}_{B}} \rho_{\mathbf{k}} e^{-\beta^{\prime} \hat{H}_{B}}, \\
M_{1}(x)=\left\langle\hat{s}_{j}^{z}\right\rangle_{0}=s B(s x),
\end{gathered}
$$

the Brillouin function

$$
B(x)=\frac{2 s+1}{2 s} \operatorname{coth}\left(\frac{2 s+1}{2 s} x\right)-\frac{1}{2 s} \operatorname{coth}\left(\frac{x}{2 s}\right) .
$$

Further, taking into account that the spin means differ from zero only when the indices numbering the particles coincide we will have from (3.2)

$$
\begin{aligned}
& K^{\nu_{1} \nu_{2}}\left(q_{1}, q_{2}\right)=\frac{1}{N} \sum_{j=1}^{N} \frac{1}{\beta^{2}} \int_{0}^{\beta} d \beta_{1} \int_{0}^{\beta} d \beta_{2} \\
& \times e^{i \beta_{1} \omega_{1}} e^{i \beta_{2} \omega_{2}} e^{-i \mathbf{k}_{1} \mathbf{r}_{j}\left(\beta_{1}\right)} e^{-i \mathbf{k}_{2} \mathbf{r}_{j}\left(\beta_{2}\right)} \\
& \times\left[\left\langle\hat{T}_{\beta} \hat{s}_{j}^{\nu_{1}}\left(\beta_{1}\right) \hat{s}_{j}^{\nu_{2}}\left(\beta_{2}\right)\right\rangle_{0}-\left\langle\hat{s}_{j}^{\nu_{1}}\right\rangle_{0}\left\langle\hat{s}_{j}^{\nu_{2}}\right\rangle_{0}\right], \\
& \hat{s}_{j}^{\nu}(\beta)=e^{\beta \hat{H}_{0}^{*}} \hat{s}_{j}^{\nu} e^{-\beta \hat{H}_{0}^{*}} .
\end{aligned}
$$

We will make here an approximation complying to the random phase approximation taken by us, viz., we will assume that we will have the main contribution from $K^{\nu_{1} \nu_{2}}\left(q_{1}, q_{2}\right)$ when

$$
e^{-i \mathbf{k}_{1} \mathbf{r}_{j}\left(\beta_{1}\right)} e^{-i \mathbf{k}_{2} \mathbf{r}_{j}\left(\beta_{2}\right)} \rightarrow \delta\left(\mathbf{k}_{1}+\mathbf{k}_{2}\right) .
$$

In this approximation from (3.6) we will find [5]:

$$
\begin{aligned}
K^{z z}\left(q_{1}, q_{2}\right) & =M_{2}(x) \delta\left(\mathbf{k}_{1}+\mathbf{k}_{2}\right) \delta\left(\omega_{1}\right) \delta\left(\omega_{2}\right), \\
M_{2}(x) & =\frac{d M_{1}(x)}{d x}, \\
K^{+-}\left(q_{1}, q_{2}\right) & =2 M_{1}(x) K_{\omega_{1}} \delta\left(\mathbf{k}_{1}+\mathbf{k}_{2}\right) \delta\left(\omega_{1}+\omega_{2}\right), \\
K_{\omega} & =\frac{1}{x+i \beta \omega}, \\
K^{-+}\left(q_{1}, q_{2}\right) & =K^{+-}\left(q_{1}, q_{2}\right) .
\end{aligned}
$$

The remaining quantities $K^{\nu_{1} \nu_{2}}\left(q_{1}, q_{2}\right)$ equal zero.

Substituting expression (3.1) in (2.16) and taking into account (3.3) and (3.7) we obtain after integrating over the variables $\varphi_{q}^{x}, \varphi_{q}^{y}$ : 


$$
\begin{aligned}
\operatorname{Sp}_{s} e^{-\beta \hat{H}} & =e^{-\beta \hat{H}_{B}} \exp \left(-\beta \frac{N s(s+1)}{2 V} \sum_{\mathbf{k}} J_{k}\right) \sqrt{\frac{N}{2 \pi}} \int_{-\infty}^{\infty} d \eta e^{-\beta F_{0}} \exp \left[-\sum_{q} \ln \left(1-\beta \frac{N}{V} J_{k} M_{1}(x) K_{\omega}\right)\right] \\
& \times \prod_{q}^{\prime} \int_{-\infty}^{\infty} \frac{d \varphi_{q}^{z^{\prime}}}{\sqrt{\pi}} \int_{-\infty}^{\infty} \frac{d \varphi_{q}^{z^{\prime \prime}}}{\sqrt{\pi}} e^{-\frac{1}{2} \sum_{q}^{\prime} \varphi_{q}^{z} \varphi_{-q}^{z}} \\
& \times \hat{T}_{\beta} \exp \left[\sum_{q}^{\prime} \varphi_{q}^{z} \sqrt{\beta \frac{N}{V} J_{k}} M_{1}(x) \frac{1}{\beta} \int_{0}^{\beta} d \beta^{\prime} e^{i \beta^{\prime} \omega} \rho_{\mathbf{k}}\left(\beta^{\prime}\right)+\frac{1}{2} \sum_{\mathbf{k} \neq 0} \beta \frac{N}{V} J_{k} M_{2}(x) \varphi_{\mathbf{k}}^{z} \varphi_{-\mathbf{k}}^{z}\right],
\end{aligned}
$$

here $\varphi_{\mathbf{k}}=\varphi_{q}^{z}$ at $q=(\mathbf{k}, 0)$.

We will preserve in the index of the operator exponent in (3.8) only the members from $q=0$ and remarking that

$$
e^{-\beta \hat{H}_{B}} \hat{T}_{\beta} \exp \left[\sum_{\mathbf{k} \neq 0} \varphi_{\mathbf{k}}^{z} \sqrt{\beta \frac{N}{V} J_{k}} M_{1}(x) \frac{1}{\beta} \int_{0}^{\beta} d \beta^{\prime} \rho_{\mathbf{k}}\left(\beta^{\prime}\right)\right]=\exp \left[-\beta \hat{H}_{B}+\sum_{\mathbf{k} \neq 0} \varphi_{\mathbf{k}}^{z} \sqrt{\beta \frac{N}{V} J_{k}} M_{1}(x) \rho_{\mathbf{k}}\right],
$$

we carry out integration by the variables $\varphi_{q}^{z}$, taking into account the fact that the operators $\hat{H}_{B}$ and $\rho_{\mathbf{k}}$ do not commute. Remaining in the framework of the adopted random phase approximation we find:

$$
\mathrm{Sp}_{s} e^{\beta \hat{H}}=\sqrt{\frac{N}{2 \pi}} \int_{-\infty}^{\infty} d \eta e^{-\beta F_{\mathrm{s}}} e^{-\beta \hat{H}_{B}^{*}},
$$

which depends on the $\eta$ parameter free energy of the spin subsystem (in the thermodynamic limit $N \rightarrow \infty$, $V \rightarrow \infty, N / V=$ const)

$$
\begin{aligned}
F_{s} & =F_{0}+\frac{N s(s+1)}{2 V} \sum_{\mathbf{k}} J_{k} \\
& +\frac{1}{\beta} \sum_{\mathbf{k}} \sum_{\omega} \ln \left[1-\beta \frac{N}{V} J_{k} M_{1}(x) K_{\omega}\right] \\
& +\frac{1}{2 \beta} \sum_{\mathbf{k} \neq 0} \ln \left[1-\beta \frac{N}{V} J_{k} M_{2}(x)\right]
\end{aligned}
$$

which after being summed by the frequencies $\omega$ looks as follows [5]:

$$
\begin{aligned}
F_{s} & =F_{0}+\frac{N s(s+1)}{2 V} \sum_{\mathbf{k}} J_{k} \\
& +\frac{1}{\beta} \sum_{\mathbf{k}} \ln \left[\sinh \left(\frac{x-\beta \frac{N}{V} J_{k} M_{1}(x)}{2}\right) / \sinh \frac{x}{2}\right] \\
& +\frac{1}{2 \beta} \sum_{\mathbf{k} \neq 0} \ln \left[1-\beta \frac{N}{V} J_{k} M_{2}(x)\right] .
\end{aligned}
$$

The operator $\hat{H}_{B}^{*}$ in (3.9) is the effective liquid Hamilto- nian

$$
\hat{H}_{B}^{*}=\hat{H}_{B}-\sum_{\mathbf{k}} \frac{N}{2 V} J_{k} \frac{M_{1}^{2}(x)}{1-\beta \frac{N}{V} J_{k} M_{2}(x)} \rho_{\mathbf{k}} \rho_{-\mathbf{k}} .
$$

Otherwise stated, by taking into consideration (2.2)

$$
\begin{aligned}
\hat{H}_{B}^{*} & =\sum_{j=1}^{N} \frac{\hat{\mathbf{p}}^{2}}{2 m}+\frac{N(N-1)}{2 V} \nu_{0} \\
& -\frac{N}{2 V} \sum_{\mathbf{k} \neq 0} \nu_{k}+\frac{N}{2 V} \sum_{\mathbf{k} \neq 0} \nu_{k}^{*} \rho_{\mathbf{k}} \rho_{-\mathbf{k}},
\end{aligned}
$$

where renormalized by the longitudinal statistical spin correlations the Fourier coefficient (2.3) of the potential energy interaction

$$
\nu_{k}^{*}=\nu_{k}-J_{k} \frac{M_{1}^{2}(x)}{1-\beta \frac{N}{V} J_{k} M_{2}(x)} .
$$

Let us return to expression (3.8). If we take into consideration in the operator exponent index the members with $\varphi_{q \neq 0}^{z}$, we will see that what results is an essentially quantum-dynamic effect. Indeed, for the classical liquid when $\hbar \rightarrow 0$ the operators $\hat{H}_{B}$ and $\rho_{k}$ commute and the quantity $\rho_{\mathbf{k}}\left(\beta^{\prime}\right) \rightarrow \rho_{\mathbf{k}}$. After this the integral over $\beta^{\prime}$ will require $\omega=0$ which contradicts the condition which is imposed on the summation by $q \neq 0$. Thus, the contribution of these members into (3.8) in the classical case equals zero.

The calculation of these quantities is to be conducted taking into account the renormalized liquid Hamiltonian effect. We will write the result after integration over the variables $\varphi_{\mathbf{q} \neq 0}^{z}$ staying in the same approximation in which we obtained expression (3.9) from (3.8): 


$$
\begin{aligned}
& \operatorname{Sp}_{s} e^{-\beta \hat{H}}=\sqrt{\frac{N}{2 \pi}} \int_{-\infty}^{\infty} d \eta e^{-\beta F_{s}} e^{-\beta \hat{H}_{B}^{*}} \hat{T}_{B} \exp \left\{\frac{1}{2} \sum_{q \neq 0} \beta \frac{N}{V} J_{k} M_{1}^{2}(x)\left|\frac{1}{\beta} \int_{0}^{\beta} d \beta^{\prime} e^{i \beta^{\prime} \omega} \tilde{\rho}_{\mathbf{k}}\left(\beta^{\prime}\right)\right|^{2}\right\}, \\
& \tilde{\rho}_{\mathbf{k}}\left(\beta^{\prime}\right)=e^{\beta^{\prime} \hat{H}_{B}^{*}} \rho_{\mathbf{k}} e^{-\beta^{\prime} \hat{H}_{B}^{*}}
\end{aligned}
$$

\section{FREE ENERGY}

In compliance with designation (2.1) the full partition function $Z$ can be obtained by tracing the operator (3.9) or (3.13) over to the liquid degrees of freedom. In this section the calculus of $Z$ is carried out without taking into account the quantum-dynamic effects making use of formula (3.9):

$$
Z=\sqrt{\frac{N}{2 \pi}} \int_{-\infty}^{\infty} d \eta e^{-\beta F_{s}} Z_{B}
$$

where the partition function of the liquid subsystem with the renormalized interparticle interaction

$$
Z_{B}=\mathrm{Sp} e^{-\beta \hat{H}_{B}^{*}}
$$

Up till now the statistics of particles was of no effect whatsoever in our calculations. Now, when calculating the partition function $Z_{B}$ we take into account the fact that the particle abides by the Bose-Einstein statistics and make use for this quantity the expression found in $[7]$

$$
\begin{aligned}
Z_{B} & =Z_{B}^{0} \exp \left\{-\beta E_{0}+\sum_{\mathbf{k} \neq 0} \ln \left(\frac{1-e^{-\beta \hbar^{2} k^{2} / 2 m}}{1-e^{-\beta E(k)}}\right)+\frac{1}{2} \sum_{\mathbf{k} \neq 0} \ln \left(\frac{\alpha_{k} \tanh \left[\frac{\beta}{2} E(k)\right]}{\tanh \left[\beta \frac{\hbar^{2} k^{2}}{4 m}\right]}\right)\right. \\
& \left.-\frac{1}{2} \sum_{\mathbf{k} \neq 0} \ln \left[1+S_{0}(k)\left(\alpha_{k} \tanh \left[\frac{\beta}{2} E(k)\right]-\tanh \left[\beta \frac{\hbar^{2} k^{2}}{4 m}\right]\right)\right]\right\},
\end{aligned}
$$

where the partition function of the ideal Bose-gas

$$
Z_{B}^{0}=\exp \left[-\sum_{\mathbf{k}} \ln \left(1-z_{0} e^{-\beta \hbar^{2} k^{2} / 2 m}\right)\right]
$$

and the structure factor

$$
\begin{aligned}
S_{0}(q) & =1+\frac{1}{N} \sum_{\mathbf{p}} n_{p} n_{|\mathbf{p}+\mathbf{q}|} \\
n_{p} & =\frac{1}{z_{0}^{-1} e^{\beta \hbar^{2} p^{2} / 2 m}-1}
\end{aligned}
$$

and the activeness (2.11) is excluded by the condition

$$
\sum_{\mathbf{p}} n_{p}=N
$$

The quantities $E_{0}$ and $E(k)$ in (4.2) are the ground-state energy and the elementary excitation spectrum of the Bose-gas in Bogoliubov's approximation [8,9]:

$$
E_{0}=\frac{N(N-1)}{2 V} \nu_{0}-\frac{N}{2 V} \sum_{\mathbf{k} \neq 0} \nu_{k}+\sum_{\mathbf{k} \neq 0} \frac{\hbar^{2} k^{2}}{4 m}\left(\alpha_{k}-1\right),
$$

$$
E(k)=\frac{\hbar^{2} k^{2}}{2 m} \alpha_{k}
$$

here the quantity

$$
\alpha_{k}=\sqrt{1+\frac{2 N}{V} \nu_{k}^{*} / \frac{\hbar^{2} k^{2}}{2 m}}
$$

The partition function (4.2) depends on the parameter $\eta$ through the renormalized interaction $\nu_{k}^{*}$ from (3.12).

Expression (4.2) shows some important properties. In the limit $\hbar \rightarrow 0$ it transforms into the well-known expression for the partition function of the classical liquid 
in the random phase approximation

$$
\begin{gathered}
Z_{B}=Z_{B}^{0} \exp \left[-\beta \frac{N(N-1)}{2 V} \nu_{0}+\frac{1}{2} \sum_{\mathbf{k} \neq 0} \beta \frac{N}{V} \nu_{k}\right. \\
\left.-\frac{1}{2} \sum_{\mathbf{k} \neq \mathbf{0}} \ln \left(1+\beta \frac{N}{V} \nu_{q}^{*}\right)\right], \\
Z_{B}^{0}=\frac{V^{N}}{N !}\left(\frac{m}{2 \pi \beta \hbar^{2}}\right)^{3 N / 2} .
\end{gathered}
$$

Thus, the classical limit in our theory reproduces the result found in [5].

At low temperatures $T \rightarrow 0$ from (4.2) we find

$$
Z_{B}=\exp \left[-\beta E_{0}-\sum_{\mathbf{k} \neq 0} \ln \left(1-e^{-\beta E(k)}\right)\right]
$$

This formula coincides with the results of Bogoliubov's theory that is working quite well in the low-temperature regime.

Let us substitute (4.20) into (4.1) and let us integrate applying the $\eta$ saddle-point method. As a result of this for the free energy

$$
F=-\frac{1}{\beta} \ln Z
$$

we find

$$
\begin{aligned}
& F=F_{s}+F_{B}, \\
& F_{B}=-\frac{1}{\beta} \ln Z_{B} .
\end{aligned}
$$

The parameter $\eta$ is eliminated from here with the help of condition

$$
\frac{d F}{d \eta}=0
$$

which is an equation for the mean magnetized system

$$
M=\mu\left\langle\sum_{j=1}^{N} \hat{s}_{j}^{z}\right\rangle
$$

where the angle brackets denote the operation of complete averaging

$$
\langle\ldots\rangle=\operatorname{Sp}\left[e^{-\beta \hat{H}}(\ldots)\right] / \operatorname{Sp} e^{-\beta \hat{H}}
$$

Indeed, should we make use for the statistical operator
(2.7) the signification which looks like a functional integral (2.10) and should we write the quantity $\sum_{j=1}^{N} \hat{s}_{j}^{z}$ as a derivative by $\varphi_{0}^{z}$ from the operator exponent in (2.10) and we further by integration shift in portions this derivative leftwards giving under the integral the factor $\sim \varphi_{0}^{z}$, we obtain

$$
M=\mu N \eta / \sqrt{\beta \frac{N}{V} J_{0}} .
$$

From this it follows that the quantity $\eta$ is an order parameter and corresponds to the rise of the magnetic ordering.

In the molecular-field approximation when we use expression (2.17) for the free energy the magnetic state equation (4.5) for the order parameter $\eta$ looks as follows:

$$
\begin{aligned}
\eta & =\sqrt{\beta \frac{N}{V} J_{0}} s B(s x), \\
x & =\beta \mu \mathcal{H}+\eta \sqrt{\beta \frac{N}{V} J_{0}} .
\end{aligned}
$$

At $\mathcal{H}=0$ for the temperature $T<T_{c}$ where

$$
T_{c}=\frac{s(s+1)}{3} \frac{N}{V} J_{0}
$$

we have the non-trivial solution of equation (4.7) for the magnetization (4.6), $\eta \neq 0$, i. e., magnetic ordering arises in the system.

The formulae arrived at in this section make it possible to calculate both fluid and magnetic characteristics if we give the model expressions for $\nu_{k}$ and $J_{k}$.

\section{THE QUANTUM-DYNAMIC CORRECTION TO THE FREE ENERGY}

Let us now calculate a correction to the free energy $\Delta F$ caused by taking into consideration the quantumdynamic effects, i. e., of the expression under $\hat{T}_{\beta}$ in (3.13). Hence, instead of equation (4.1) we will have

$$
Z=\sqrt{\frac{N}{2 \pi}} \int_{-\infty}^{\infty} d \eta e^{-\beta F_{s}} Z_{B} e^{-\beta \Delta F}
$$

where

$$
\begin{aligned}
\Delta F & =-\sum_{\mathbf{q} \neq 0} \frac{N}{V} J_{k} M_{1}^{2}(x) \frac{1}{\beta^{2}} \int_{0}^{\beta} d \beta_{1} \int_{0}^{\beta_{1}} d \beta_{2} \\
& \times e^{i\left(\beta_{1}-\beta_{2}\right) \omega}\left\langle\tilde{\rho}_{\mathbf{k}}\left(\beta_{1}-\beta_{2}\right) \rho_{-\mathbf{k}}\right\rangle_{B}
\end{aligned}
$$

and the angle brackets 


$$
\langle\ldots\rangle_{B}=\frac{\operatorname{Sp}\left[e^{-\beta \hat{H}_{B}^{*}}(\ldots)\right]}{\operatorname{Sp} e^{-\beta \hat{H}_{B}^{*}}}
$$

imply averaging over the fluid degrees of freedom with the effective Hamiltonian (3.11). This average is easily computed in the Bogoliubov approximation:

$$
\begin{aligned}
& \left\langle\tilde{\rho}_{\mathbf{k}}\left(\beta_{1}-\beta_{2}\right) \rho_{-\mathbf{k}}\right\rangle_{B}=\frac{1}{\alpha_{k}}\left[\frac{e^{\left(\beta_{1}-\beta_{2}\right) E(k)}}{e^{\beta E(k)}-1}\right. \\
& \left.+e^{-\left(\beta_{1}-\beta_{2}\right) E(k)} \frac{e^{\beta E(k)}}{e^{\beta E(k)}-1}\right] .
\end{aligned}
$$

Elementary integrating over $\beta_{1}, \beta_{2}$ in (5.2) after substituting (5.3) with subsequent summing by the frequencies $\omega \neq 0$ ultimately leads to

$$
\begin{aligned}
\Delta F & =-\frac{N}{2 V} \sum_{\mathbf{k} \neq 0} \frac{J_{k} M_{1}^{2}(x)}{\alpha_{k}} \\
& \times\left(\operatorname{coth}\left[\beta \frac{E(k)}{2}\right]-1 /\left[\beta \frac{E(k)}{2}\right]\right) .
\end{aligned}
$$

In accordance with (5.1) this quantity should be added to (4.4) in order to obtain a complete expression for the free energy in the random phase approximation.

In the classical limit $\hbar \rightarrow 0$, as can be seen from (5.4), the correction $\Delta F=0$ which has already been discussed in Section III. In the low temperatures limit

$$
\Delta F=-\frac{N}{2 V} s^{2} \sum_{\mathbf{k} \neq 0} \frac{J_{k}}{\sqrt{1+\frac{2 N}{V}\left(\nu_{k}-s^{2} J_{k}\right) / \frac{\hbar^{2} k^{2}}{2 m}}}
$$

this quantity contributing additionally to the energy of the ground state $E_{0}$ of the magnetic Bose-liquid.

\section{THE STRUCTURE FACTOR}

By definition the fluid structure factor

$$
S(q)=\left\langle\rho_{\mathbf{k}} \rho_{-\mathbf{k}}\right\rangle
$$

is reduced to calculating the average for a system with the effective Hamiltonian (3.11) and according to [7] equals

$$
S(k)=\frac{S_{0}(k)}{1+S_{0}(k)\left(\alpha_{k} \tanh [\beta E(k) / 2]-\tanh \left[\beta \hbar^{2} k^{2} / 4 m\right]\right)} .
$$

At the absolute zero of temperature or in a strong external field $\mathcal{H}$. It follows that taking into account the fact that the hyperbolic tangents as well as the quantity $S_{0}(k)$ are tending to unity we have

$$
S(k)=\frac{1}{\alpha_{k}}, \quad T=0
$$

and the quantity $\nu_{k}^{*}$ in the expression for $\alpha_{q}$ (4.3) in compliance with (3.12) equals

$$
\nu_{k}^{*}=\nu_{k}-s^{2} J_{k}, \quad T=0 .
$$

It is obvious that this expression follows directly from (2.2), (2.5) when the system finds itself in the magnetically ordered state and each spin has a direction of the field. The structure factor (6.2) can be rewritten as follows

$$
S(k)=\frac{S_{B}(k)}{\sqrt{1-\frac{2 N}{V} s^{2} J_{k} S_{B}^{2}(k) / \frac{\hbar^{2} k^{2}}{2 m}}}
$$

where the Bose-liquid structure factor without the consideration of the magnetic interactions

$$
S_{B}(k)=1 / \sqrt{1+\frac{2 N}{V} \nu_{k} / \frac{\hbar^{2} k^{2}}{2 m}} .
$$

As can be seen from (6.4) the switching on of the magnetic interaction increases the structure factor for the wave vectors $\mathbf{k}$ at which the Fourier coefficient of the exchange interaction energy (2.6) $J_{k}>0$ and when $J_{k}<0$ it tends to decrease it.

At $k \rightarrow 0$ from (6.4) we have a long-wave asymptotics of the structure factor

$$
S(k)=\frac{\hbar k}{2 m c}
$$

where the sound velocity

$$
c=c_{B} \sqrt{1-\frac{N}{V} s^{2} J_{0} / m c_{B}^{2}},
$$




\section{O. VAKARCHUK}

where

$$
c_{B}=\sqrt{\frac{N \nu_{0}}{V m}}
$$

is the sound velocity in the system disregarding the magnetic interactions.

Henceforth we are justified to conclude that the sound velocity in the Bose-liquid tends to decrease as magnetisation increases.

Within the classical limit $\hbar \rightarrow 0$ in formula (6.1) the structure factor of the ideal gas $S_{0}(k) \rightarrow 1$, and out of the hyperbolic tangents only the linear members of decomposition 'survive'. As a result of this the structure factor

$$
S(k)=\frac{1}{1+\beta \frac{N}{V} \nu_{q}^{*}},
$$

and taking into account (3.12)

$$
\begin{aligned}
& S(k)=S_{B}(k) /\left[1-\beta \frac{N}{V} J_{k} S_{B}(k)\right. \\
& \left.\times \frac{M_{1}^{2}(x)}{1-\beta \frac{N}{V} J_{k} M_{2}(x)}\right],
\end{aligned}
$$

where the structure factor without magnetic interactions

$$
S_{B}(k)=\frac{1}{1+\beta \frac{N}{V} \nu_{k}} .
$$

Expression (6.7) was received in [5].

Similarly to the quantum liquid $S(k)>S_{B}(k)$ for the case when $J_{k}>0$ and, conversely, when $S(k)<S_{B}(k)$ at $J_{k}<0$. The sound velocity $c$ can be found from the thermodynamic correlation $S_{q \rightarrow 0}=T / m c^{2}[10]$ :

$$
\begin{aligned}
c^{2} & =c_{B}^{2}\left[1-\frac{N}{V} \frac{J_{0}}{m c_{B}^{2}} \frac{M_{1}^{2}(x)}{1-\beta \frac{N}{V} J_{0} M_{2}(x)}\right], \\
c_{B}^{2} & =\frac{T}{m}\left(1+\beta \frac{N}{V} \nu_{0}\right) .
\end{aligned}
$$

Hence, when we switch on the magnetic field the sound velocity decreases, just as in the quantum case. It is curious that in strong fields formula (6.8) formally coincides with formula (6.6) for the quantum liquid.

\section{BOSE-CONDENSATE}

We will study the phenomenon of the Bose-Einstein condensation making use of the theory of Bose-fluids from the general expression for the quantity of Bose- condensate [11]. At the absolute zero of temperature for the relative number of the system particles whose momenta equal zero taking into account the renormalizing of the interparticle interaction we have:

$$
\frac{N_{0}}{N}=\exp \left[-\frac{1}{N} \sum_{\mathbf{k} \neq 0} \frac{\left(\alpha_{k}-1\right)^{2}}{4 \alpha_{k}}\right]
$$

as

$$
\alpha_{k}=\frac{1}{S_{B}(k)} \sqrt{1-\frac{2 N}{V} s^{2} J_{k} S_{B}^{2}(k) / \frac{\hbar^{2} k^{2}}{2 m}},
$$

and the expression for $S_{B}(k)$ is given by formula (6.5). In the linear approximation by $J_{k}$ from $(7.1)$ we find

$$
\begin{aligned}
& \frac{N_{0}}{N}=\exp \left\{-\frac{1}{N} \sum_{\mathbf{k} \neq 0} \frac{\left[S_{B}(k)-1\right]^{2}}{4 S_{B}(k)}\right. \\
& \left.+\frac{1}{4 N} \sum_{\mathbf{k} \neq 0} S_{B}(k)\left[1-S_{B}^{2}(k)\right] \frac{s^{2} N}{V} J_{k} / \frac{\hbar^{2} k^{2}}{2 m}\right\} .
\end{aligned}
$$

For the values of the wave vector giving the main contribution at the integration by $\mathbf{k}$ in $(7.2)$ the quantity $J_{k}>0$, and $S_{B}(k)<1$. That is why the second term in the index of the exponent in (7.2) is a positive quantity. Hence, the switching on of the magnetic interactions proves to increase the quantity of Bose-condensate in the magnetic Bose-liquid.

This conclusion can be maintained by a direct calculus of quantity (7.1) for the known model of hard spheres with the diameter $a$. Thus, we will assume that for all values of $k$ :

$$
\begin{aligned}
& \nu_{k}=\nu_{0}=\frac{4 \pi \hbar^{2}}{m} a, \quad J_{k}=J_{0}, \\
& \nu_{k}^{*}=\frac{4 \pi \hbar^{2} a^{*}}{m}, \quad a^{*}=a\left(1-s^{2} \frac{J_{0}}{\nu_{0}}\right) .
\end{aligned}
$$

Now, taking into account the thermodynamic boundary $V \rightarrow \infty$ we pass over to (7.1) due to the summation over the wave vector $\mathbf{k}$ to integration. This is elementary integration and as a result we have

$$
\frac{N_{0}}{N}=\exp \left[-\frac{8}{3} \sqrt{\frac{N a^{3}}{\pi V}}\left(1-s^{2} \frac{J_{0}}{\nu_{0}}\right)^{3 / 2}\right] .
$$

Thus, the magnetic interactions of the ferro-magnetic type, when $J_{k}>0$, prove to be conducive to the phenomenon of Bose-condensation.

A study of the temperature dependence of the Bosecondensate quantity just as other thermodynamic and 
structure functions of the magnetic atoms quantum liquid on the basis of the established formulae calls for a modelling of the interparticle interactions. This issue will be presented separately.
[1] W. C. Stwalley, L. H. Nosanow, Phys. Rev. Lett. 36, 910 (1976).

[2] M. Edwards, R. J. Dodd, C. W. Clark, K. Burnett, J. Res. Natl. Inst. Stand. Technol. 101, 553 (1996).

[3] D. G. Fried, T. C. Killian, L. Willmann, D. Landhuis, S. C. Moss, D. Kleppner, T. J. Greytak, Phys. Rev. Lett. 81, 3811 (1998).

[4] I. O. Vakarchuk, in Proceeding of Workshop on Modern Problems of Soft Matter Theory, 27-31 August 2000, Lviv, Ukraine. Book of Abstract (Lviv, 2000), p. 26.

[5] I. A. Vakarchuk, Yu. K. Rudavskii, G. V. Ponedilok, Teor. Mat. Fiz. 58, 445 (1984) [Theor. Math. Phys. 58, 291 (1984)].

[6] I. A. Vakarchuk, Yu. K. Rudavskii, Teor. Mat. Fiz. 49,
234 (1981) [Theor. Math. Phys. 49, 1002 (1981)].

[7] I. O. Vakarchuk, J. Phys. Stud. (in press).

[8] N. N. Bogoliubov, J. Phys. USSR 9, 23 (1947).

[9] N. N. Bogolyubov, D. N. Zubarev, Zh. Eksp. Teor. Fiz. 28, 129 (1955).

[10] I. O. Vakarchuk, Introduction into the Many-Body Problem (Lviv University Press, Lviv, 1999) [in Ukrainian].

[11] I. O. Vakarchuk, Teor. Mat. Fiz. 23, 260 (1975) [Theor. Math. Phys. 23, 496 (1975)]; Teor. Mat. Fiz. 65, 285 (1985) [Theor. Math. Phys. 65, 1164 (1985)]; Teor. Mat. Fiz. 80, 439 (1989) [Theor. Math. Phys. 80, 983 (1989)]; Teor. Mat. Fiz. 82, 438 (1990) [Theor. Math. Phys. 82, $308(1990)]$.

\title{
МАГНЕТНА БОЗЕ-РІДИНА
}

\author{
I. О. Вакарчук \\ Львівсъкий начіональний університет імені Івана Франка, кафедра теоретичной фізики \\ вул. Драгоманова, 12, Львів, 79005, Украӥна
}

\footnotetext{
Досліджено квантову рідину, яка складається з бозе-частинок, що мають магнетний момент. Обчислено термодинамічні та структурні функпї такої системи в наближенні хаотичних фаз за допомогою методу функџіонального інтегрування. Отримані вирази є справедливими як у низькотемпературній ділянці, так i в квазікласичній гранищі, де вони відтворюють відомі результати теорії класичних магнетних рідин, які ми знайшли раніше. Виписано рідинні та магнетні рівняння стану та досліджено вплив зовнішнього магнетного поля на явище бозе-айнштайнівської конденсапії.
} 